HD-THEP-94-40

\title{
Fermionic Coset Realization of the Critical Ising Model
}

\author{
D. C. Cabraf and K. D. Rothe \\ Institut für Theoretische Physik \\ Universität Heidelberg \\ Philosophenweg 16, D-69120 Heidelberg
}

\begin{abstract}
We obtain an explicit realization of all the primary fields of the Ising model in terms of a conformal field theory of constrained fermions. The four-point correlators of the energy, order and disorder operators are explicitly calculated.
\end{abstract}

* On leave of absence from the Universidad Nacional de La Plata, Argentina 
The quantum field theoretic description of critical statistical systems has received much attention in the past [1]. In particular, the Ising model has provided a useful laboratory for testing field-theoretical ideas and techniques. It has been known for some time that at criticality it can be described by free massless Majorana fermions $\psi, \bar{\psi}[2,3]$. In terms of these, the energy operator $\epsilon(x)$ is given by the local product $\psi \bar{\psi}$. Using this representation, multicorrelators of the energy operator have been computed [3]. Some correlators involving the order operator $\sigma(x)$ and disorder operator $\mu(x)$ have also been calculated using indirect methods. The reason is that a local representation in terms of Majorana fermions is lacking in this case.

On the other hand, the Ising model can be described in terms of a Conformal Quantum Field Theory (CQFT) of central charge $c=\frac{1}{2}$ [4], corresponding to $k=1$ in the minimal unitary series [5]

$$
c=1-\frac{6}{(k+2)(k+3)}
$$

Using conformal methods, four-point functions involving the order and disorder operators have been calculated [4] on the basis of representation theory of the Virasoro algebra. In this approach, $\sigma(x)$ and $\mu(x)$ are regarded as primaries of conformal dimensions $\left(\frac{1}{16}, \frac{1}{16}\right)$, with correlators satisfying the corresponding null-vector equation.

In this letter we use the ideas of CQFT and of ref. [6], [7] in order to obtain a fermionic coset realization of the critical Ising model. In this way we arrive at a complete description of the model, and in particular of the order and disorder operators in terms of local products of the fundamental observables of the theory.

The Ising model can be described by a conformal quantum field theory of central charge $c=\frac{1}{2}$ [5] and corresponds to a coset model $\mathrm{G} / \mathrm{H}$ with 
$G=S U(2)_{1} \times S U(2)_{1}$ and $H=S U(2)_{2}$ [8]. In the fermionic description [6], [7] this theory can be realized by making the identification

$$
\frac{S U(2)_{1} \times S U(2)_{1}}{S U(2)_{2}}=\frac{\frac{U(2)}{U(1)} \times \frac{U(2)}{U(1)}}{S U(2)_{2}}
$$

The corresponding Lagrangian is obtained by starting from two different kinds of $U(2)$-fermions, and gauging the respective $U(1)$ subgroups, as well as the diagonal subgroup $S U(2)$ :

$$
\begin{aligned}
\mathcal{L}= & \frac{1}{\sqrt{2} \pi} \bar{\psi}^{i}\left((\not \partial+i \not \phi) \delta_{i j}+i A_{i j}\right) \psi^{j} \\
& +\frac{1}{\sqrt{2} \pi} \bar{\chi}^{i}\left((\not \partial+i \not b) \delta_{i j}+i A_{i j}\right) \chi^{j}
\end{aligned}
$$

where $i, j=1,2$ and $A_{\mu}$ are $S U(2)$ Lie-algebra-valued fields.

We now make the change of variables [9]

$$
\begin{aligned}
& a=i\left(\bar{\partial} h_{1}\right) h_{1}^{-1}, \bar{a}=i\left(\partial \bar{h}_{1}\right) \bar{h}_{1}^{-1} \\
& b=i\left(\bar{\partial} h_{2}\right) h_{2}^{-1}, \quad \bar{b}=i\left(\partial \bar{h}_{2}\right) \bar{h}_{2}^{-1} \\
& A=i(\bar{\partial} g) g^{-1}, \quad \bar{A}=i(\partial \bar{g}) \bar{g}^{-1} \\
& \psi_{1}=h_{1} g \psi_{1}^{(0)}, \quad \psi_{2}^{\dagger}=\psi_{2}^{(0) \dagger}\left(h_{1} g\right)^{-1} \\
& \psi_{2}=\bar{h}_{1} \bar{g} \psi_{2}^{(0)}, \quad \psi_{1}^{\dagger}=\psi_{1}^{(0) \dagger}\left(\bar{h}_{1} \bar{g}\right)^{-1} \\
& \chi_{1}=h_{2} g \chi_{1}^{(0)}, \quad \chi_{2}^{\dagger}=\chi_{2}^{(0) \dagger}\left(h_{2} g\right)^{-1} \\
& \chi_{2}=\bar{h}_{2} \bar{g} \chi_{2}^{(0)}, \quad \chi_{1}^{\dagger}=\chi_{1}^{(0) \dagger}\left(\bar{h}_{2} \bar{g}\right)^{-1}
\end{aligned}
$$

Taking account of the Jacobians of the respective transformations [10, 11] (see also [7]) one arrives at a decoupled form for the partition function associated with the Lagrangian (3):

$$
Z_{\frac{S U(2)_{1} \times S U(2)_{1}}{S U(2)_{2}}}=Z_{F} Z_{B} Z_{W Z W} Z_{g h}
$$

where 


$$
\begin{gathered}
Z_{F}=\int \mathcal{D} \psi^{(0)} \mathcal{D} \bar{\psi}^{(0)} \mathcal{D} \chi^{(0)} \mathcal{D} \bar{\chi}^{(0)} \exp \left(-\frac{1}{\pi} \int\left(\psi_{2}^{\dagger} \bar{\partial} \psi_{1}+\psi_{1}^{\dagger} \partial \psi_{2}\right)\right) \\
\times \exp \left(-\frac{1}{\pi} \int\left(\chi_{2}^{\dagger} \bar{\partial} \chi_{1}+\chi_{1}^{\dagger} \partial \chi_{2}\right)\right. \\
Z_{B}=\int \mathcal{D} \phi_{1} \mathcal{D} \phi_{2} \exp \left(\frac{1}{\pi} \int \phi_{1} \Delta \phi_{1}\right) \exp \left(\frac{1}{\pi} \int \phi_{2} \Delta \phi_{2}\right) \\
Z_{W Z W}=\int \mathcal{D} \tilde{g} \exp (-k W[\tilde{g}])
\end{gathered}
$$

and $Z_{g h}$ is the partition function of ghosts, whose explicit form will not be required. The fields $\phi_{1}$ and $\phi_{2}$ are defined via

$$
h_{i}=e^{-\left(\phi_{i}+i \eta_{i}\right)}, \bar{h}_{i}=e^{\left(\phi_{i}-i \eta_{i}\right)}
$$

where $\eta_{1}$ and $\eta_{2}$ are pure gauge degrees of freedom. In arriving at (5)-(8), $\eta_{1}$ and $\eta_{2}$, as well as the gauge-variant combinations $\hat{g}=g \bar{g}$ have been integrated out. $W[\tilde{g}]$ in $(8)$ is the Wess-Zumino-Witten (WZW) action [12]

$$
\begin{aligned}
W[\tilde{g}]= & -\frac{1}{8 \pi} \int d^{2} x \operatorname{tr}\left(\partial_{\mu} \tilde{g} \partial^{\mu} \tilde{g}^{-1}\right) \\
& +\frac{1}{12 \pi} \int d^{3} y \epsilon_{i j k} \operatorname{tr}\left(\tilde{g}^{-1} \partial_{i} \tilde{g} \tilde{g}^{-1} \partial_{j} \tilde{g} \tilde{g}^{-1} \partial_{k} \tilde{g}\right)
\end{aligned}
$$

where $\tilde{g}$ is defined by

$$
\tilde{g}=g^{-1} \bar{g}
$$

and $k=-\left(2+2 c_{V}\right)=-6$ is the level of the Kac Moody algebra. The negative level WZW sector is non-unitary. However, the BRST quantization condition connects the different sectors (decoupled at the Lagrangian level) and ensures unitarity [13], 14]. The central charge obtained by adding the individual contributions $c_{F}=4, c_{B}=2, c_{W Z W}=9 / 2$ and $c_{g h}=-10$ is equal to $c=1 / 2$ in accordance with the central charge of the Ising model.

The observables of the theory defined by the Lagrangian (3) will be constructed from the gauge-invariant operator products 


$$
\begin{aligned}
& \hat{\psi}^{i}(x)=e^{-i \int_{x}^{\infty} d z_{\mu} a_{\mu}}\left(P e^{-i \int_{x}^{\infty} d z^{\mu} A_{\mu}}\right)_{i j} \psi^{j}(x) \\
& \hat{\chi}^{i}(x)=e^{-i \int_{x}^{\infty} d z_{\mu} b_{\mu}}\left(P e^{-i \int_{x}^{\infty} d z^{\mu} A_{\mu}}\right)_{i j} \chi^{j}(x)
\end{aligned}
$$

As we now show, all the primaries of the Ising model can be obtained as local products of the operators (12) in the isospin zero sector. In this sector the non-abelian Schwinger line integrals associated with the diagonal $S U(2)$ gauge group will not contribute.

We begin by identifying the energy operator $\epsilon$ and order operator $\sigma$ with isospin neutral local operator products of the fundamental fields (12):

$$
\begin{aligned}
& \epsilon=\left(\left(\hat{\psi}_{2}^{\dagger} \hat{\chi}_{1}+\hat{\chi}_{2}^{\dagger} \hat{\psi}_{1}\right)\left(\hat{\chi}_{1}^{\dagger} \hat{\psi}_{2}+\hat{\psi}_{1}^{\dagger} \hat{\chi}_{2}\right)\right) \\
&=\left(\psi_{2}^{(0) \dagger} \chi_{1}^{(0)}\right)\left(\chi_{1}^{(0) \dagger} \psi_{2}^{(0)}\right) e^{2 \phi_{1}} e^{-2 \phi_{2}} \\
&+\left(\psi_{2}^{(0) \dagger} \chi_{1}^{(0)}\right)\left(\psi_{1}^{(0) \dagger} \chi_{2}^{(0)}\right) e^{\varphi_{1}-\bar{\varphi}_{1}} e^{-\left(\varphi_{1}-\bar{\varphi}_{2}\right)} \\
&+\left(\psi \leftrightarrow \chi, \quad \varphi_{1} \leftrightarrow \varphi_{2}, \quad \bar{\varphi}_{1} \leftrightarrow \bar{\varphi}_{2}\right) \\
& \sigma=\left(\hat{\psi}^{\dagger} \hat{\psi}+\hat{\chi}^{\dagger} \hat{\chi}\right) \\
&=\left(\psi_{2}^{(0) \dagger} \tilde{g} \psi_{2}^{(0)}\right) e^{2 \phi_{1}}+\left(\psi_{1}^{(0) \dagger} \tilde{g}^{-1} \psi_{1}^{(0)}\right) e^{-2 \phi_{1}} \\
&+\left(\psi \rightarrow \chi, \phi_{1} \rightarrow \phi_{2}\right) \\
& \varphi_{i}=\phi_{i}+i \int_{x}^{\infty} d z_{\mu} \epsilon_{\mu \nu} \partial_{\nu} \phi_{i} \\
& \bar{\varphi}_{i}=\phi_{i}-i \int_{x}^{\infty} d z_{\mu} \epsilon_{\mu \nu} \partial_{\nu} \phi_{i}
\end{aligned}
$$

are the holomorphic and antiholomorphic components of the fields $\phi_{i}$ and where we have identified the normal-ordered products $: g g^{-1}:$ and $: \bar{g} \bar{g}^{-1}$ : with the identity operator. The relative signs in (13) and (14) have been chosen such as to conform to the operator product expansions in the literature 
[15]. The conformal dimension of the fields can be read off from the two-point functions [16]

$$
\begin{aligned}
& \left\langle\psi_{1}^{(0)^{i_{1}}}(1) \psi_{2}^{(0)^{i_{2} \dagger}}(2)\right\rangle=\frac{1}{2} \frac{\delta^{i_{1} i_{2}}}{z_{12}} \\
& \left\langle\psi_{2}^{(0)^{j_{1}}}(1) \psi_{1}^{(0)^{j_{2}} \dagger}(2)\right\rangle=\frac{1}{2} \frac{\delta^{j_{1} j_{2}}}{\bar{z}_{12}} \\
& \left\langle e^{\alpha \varphi(1)} e^{-\alpha \varphi(2)}\right\rangle=\left(\mu z_{12}\right)^{\frac{\alpha^{2}}{8}} \\
& \left\langle e^{\alpha \bar{\varphi}(1)} e^{-\alpha \bar{\varphi}(2)}\right\rangle=\left(\mu \bar{z}_{12}\right)^{\frac{\alpha^{2}}{8}} \\
& \left\langle g^{i_{1} j_{1}}(1) g^{-1 j_{2} i_{2}}(2)\right\rangle=\left|\mu z_{12}\right|^{3 / 4} \delta^{i_{1} i_{2}} \delta^{j_{1} j_{2}}
\end{aligned}
$$

where $z_{i j}=z_{i}-z_{j}, \bar{z}_{i j}=\bar{z}_{i}-\bar{z}_{j}$. The conformal dimensions of $\epsilon$ and $\sigma$ are obtained by adding the dimensions of the components making up (13) and (14), and are found to be $h_{\epsilon}=\bar{h}_{\epsilon}=\frac{1}{2}$ and $h_{\sigma}=\bar{h}_{\sigma}=\frac{1}{16}$, respectively.

From (13) we see that all multipoint correlation functions of $\epsilon$ can be calculated in terms of the free-fermion and vertex two-point functions. Special attention has to be payed to the fact that with the definitions (15), we have

$$
\left\langle e^{\alpha \varphi(1)} e^{\beta \bar{\varphi}(2)}\right\rangle=e^{i \alpha \beta \frac{\pi}{4}}
$$

For the four-point function of $\epsilon$ one thus obtains

$$
\langle\epsilon(1) \epsilon(2) \epsilon(3) \epsilon(4)\rangle=\frac{1}{\left|z_{12} z_{34}\right|^{2}} \frac{\left|1-x+x^{2}\right|}{|1-x|^{2}}=\left|P_{f}\left(\frac{1}{z_{i j}}\right)\right|^{2}
$$

where

$$
x=\frac{z_{12} z_{34}}{z_{14} z_{32}}, \quad \bar{x}=\frac{\bar{z}_{12} \bar{z}_{34}}{\bar{z}_{14} \bar{z}_{32}} .
$$

Expression (18) agrees with the expected result [3].

The computation of the four-point function of the $\sigma$-field requires the knowledge of the four-point functions 


$$
\begin{aligned}
& G(1,2,3,4):=\left\langle\operatorname{tr}\left(\tilde{g}(1) \tilde{g}^{-1}(2)\right) \operatorname{tr}\left(\tilde{g}(3) \tilde{g}^{-1}(4)\right)\right\rangle \\
& \hat{G}(1,2,3,4):=\left\langle\operatorname{tr}\left(\tilde{g}(1) \tilde{g}^{-1}(2) \tilde{g}(3) \tilde{g}^{-1}(4)\right)\right\rangle
\end{aligned}
$$

of the level (-6) WZW field, and those obtained by permutation of the arguments. These functions can be directly extracted from the results of ref. [17]. However, for their computation it is advantageous to make use of the equivalence between the $S U(2)_{2}$ WZW theory and the fermionic coset model $U(4) /\left(S U(2)_{2} \times U(1)\right) 18$. For the particular arrangement of the arguments $(1,2,4,3)$ one arrives at the particularly simple expressions

$$
\begin{aligned}
& G(1,2,4,3)=8\left|z_{14} z_{23}\right|^{3 / 4} \frac{|x|}{|x(1-x)|^{1 / 4}}\left(f_{1}(x) f_{1}(\bar{x})+f_{2}(x) f_{2}(\bar{x})\right) \\
& \hat{G}(1,2,4,3)=-8\left|z_{14} z_{23}\right|^{3 / 4} \frac{\sqrt{x(1-\bar{x})}}{|x(1-x)|^{1 / 4}}\left(f_{1}(x) f_{2}(\bar{x})-f_{2}(x) f_{1}(\bar{x})\right)(21
\end{aligned}
$$

where

$$
f_{1}(x)=\sqrt{1+\sqrt{x}}, \quad f_{2}(x)=\sqrt{1-\sqrt{x}}
$$

From here the corresponding expressions for permutations of the arguments are easily computed. Note that the functions $f_{i}$ are the independent solutions of the hypergeometric equation arising from the null-vector equation for a primary field of conformal dimension 1/16 [4].

One can show after a lengthy, but straightforward calculation that the four-point correlator of the order operator (14) is given by the expression

$$
\begin{aligned}
& \langle\sigma(1) \sigma(2) \sigma(3) \sigma(4)\rangle= \\
& \frac{1}{\left|z_{14} z_{23}\right|^{1 / 4}} \frac{1}{|x(1-x)|^{1 / 4}}(\sqrt{1+\sqrt{x}} \sqrt{1+\sqrt{\bar{x}}}+\sqrt{1-\sqrt{x}} \sqrt{1-\sqrt{\bar{x}}}),
\end{aligned}
$$

which agrees with that obtained by BPZ [4] using general conformal arguments. (In order to compare with the BPZ result we note that expression (23) 
is invariant under any permutation of the indices, and in particular under $z_{3} \leftrightarrow z_{4}$, implying $x \rightarrow y=\frac{x}{x-1}$.)

The disorder operator should satisfy the equal-time dual algebra [19]

$$
\sigma(1) \mu(2)=e^{i \pi \theta\left(x_{1}-x_{2}\right)} \mu(2) \sigma(1)
$$

This leads us to make the following ansatz in terms of the gauge-invariant fermion fields (12)

$$
\begin{aligned}
\mu= & \hat{\psi}^{\dagger} \hat{\chi}+\hat{\chi}^{\dagger} \hat{\psi} \\
= & \left(\psi_{2}^{\dagger(0)} \tilde{g} \chi_{2}^{(0)}\right) e^{\varphi_{1}+\bar{\varphi}_{2}}+\left(\chi_{1}^{\dagger(0)} \tilde{g}^{-1} \psi_{1}^{(0)}\right) e^{-\left(\varphi_{1}+\bar{\varphi}_{2}\right)} \\
& +\left(\psi \leftrightarrow \chi, \varphi_{1} \leftrightarrow \varphi_{2}, \bar{\varphi}_{1} \leftrightarrow \bar{\varphi}_{2}\right) .
\end{aligned}
$$

Using (15) one checks that this operator has dimension 1/16, as required. With the aid of the (euclidean) equal-time commutators:

$$
\begin{aligned}
{[\varphi(1), \varphi(2)]_{E T} } & =-\frac{i \pi}{2} \operatorname{sgn}\left(x_{12}\right) \\
{[\varphi(1), \bar{\varphi}(2)]_{E T} } & =\frac{i \pi}{2} \operatorname{sgn}\left(x_{12}\right) \\
{[\varphi(1), \bar{\varphi}(2)]_{E T} } & =\frac{i \pi}{2}
\end{aligned}
$$

one furthermore checks that the order-disorder algebra (24) is satisfied.

The evaluation of the four-point correlator of the disorder operator parallels the one for the order operator, the result being again given by the r.h.s. of eq. (23), as it should be 20].

The calculation of the mixed order-disorder correlator $\langle\sigma(1) \mu(2) \sigma(3) \mu(4)\rangle$ proceeds along similar lines, and only involves the WZW four-point function in the combinations (20). Also due account has to be taken of the phases arising from (17). A straightforward calculation shows that the four-point functions arising from the individual terms in the expressions (14) and (25) for $\sigma(x)$ and $\mu(x)$, respectively, though seemingly different at first sight, have 
all the same form, and in fact contribute with the same weight to the $\sigma-\mu$ correlator. The result of the calculation is most conveniently expressed in terms of the variable $y=\frac{x}{x-1}$, and takes the form

$\left\langle\sigma(1) \mu(2) \sigma(3) \mu(4)=\frac{1}{\left|z_{13} z_{24}\right|^{1 / 4}} \frac{1}{|y(1-y)|^{1 / 4}}(\sqrt{1+\sqrt{y}} \sqrt{1-\sqrt{y}}-\sqrt{1-\sqrt{y}} \sqrt{1+\sqrt{y}})\right.$

which agrees with the result obtained by BPZ on the basis of general conformal considerations, giving further support to our ansätze.

To complete our discussion of the Ising model, we give a realization of the Onsager fermions $\psi(x)$ and $\bar{\psi}(x)$ [2], [3] in the fermionic coset framework. They are naturally identified with the gauge-invariant composites

$$
\begin{aligned}
& \psi=\hat{\psi}_{2}^{\dagger} \hat{\chi}_{1}+\hat{\chi}_{2}^{\dagger} \hat{\psi}_{1} \\
& \bar{\psi}=\hat{\psi}_{1}^{\dagger} \hat{\chi}_{2}+\hat{\chi}_{1}^{\dagger} \hat{\psi}_{2}
\end{aligned}
$$

of dimensions $\left(\frac{1}{2}, 0\right)$ and $\left(0, \frac{1}{2}\right)$, respectively.

Recalling (13) we see that the assignment (28) is in agreement with the usual representation of the energy operators in terms of Majorana fermions [3]. Note that they have the expected property $\psi^{\dagger}=\bar{\psi}$.

Our explicit calculations lend support to the general validity of our fermionic coset realization of all the primary operators of the critical Ising model in terms of local products of BRST-invariant fermionic fields. In the framework of Majorana fermions, this is not possible. Similarly, the bosonic coset description [21] does not lend itself to a natural identification of all the primaries of the Ising model.

In our fermionic coset formulation of the Ising model the identification became possible at the price of allowing for an underlying non-abelian structure realized by WZW fields, free massless fermions and bosons. In particular the primaries of the Ising model have been represented in terms of local BRST 
invariant products of free Dirac fermions, vertex operators of free massless bosons, and negative-level WZW fields. Thus the calculation of multipoint correlators of the primaries is only restricted by our lack of knowledge of $n$-point correlators of WZW fields, with $n>4$. It interesting to note, that according to (14) the application of an external magnetic field in the Ising model correspond to the addition of a mass term in the fermionic coset formulation. On the other hand, an off-critical perturbation linear in the energy operator is described acccording to (13) by a quartic self-interaction of fermions.

Our construction for the Ising model can be generalized to other statistical models corresponding to the minimal unitary series, with the Ising model corresponding to $k=1$. Work in this direction will be reported elsewhere.

Our construction may also prove useful for studying perturbations of minimal models about the critical point. The same applies to the study of statistical systems in the presence of line defects [22], [23].

Acknowledgement: One of the authors (D.C.C.) would like to thank the Commission of the European Community for the "Marie Curie Fellowship", which made this collaboration possible. We also thank Jürgen Fuchs for a useful discussion.

\section{References}

[1] C. Itzykson, H. Saleur, and J.-B. Zuber, Editors, "Conformal Invariance and Applications to Statistical Mechanics", World Scientific 1988.

[2] L. Onsager, Phys. Rev. 65, 117 (1944). 
[3] M. Bander and C. Itzykson, Phys. Rev. D15, 463 (1977);

C. Itzykson, and J. M. Drouffe, Statistical Field Theory, Vol. 1,2, Cambridge Univ. Press 1989.

[4] A. A. Belavin, A. M. Polyakov, and A. B. Zamolodchikov, Nucl. Phys. B241, 333 (1981).

[5] D. Friedan, Z. Qiu, and S. Shenker, Phys. Rev. Lett. 52, 1575 (1984).

[6] E. Bardacki, E. Rabinovici, and B. Saring, Nucl. Phys. B229, 151 (1988).

[7] D. Cabra, E. Moreno, and C. von Reichenbach, Int. J. Mod. Phys. A5, 2313 (1990).

[8] P. Goddard, A. Kent, and D. Olive, Int. J. Mod. Phys. A1, 303 (1986).

[9] Our notation is $a=\frac{1}{\sqrt{2}}\left(a_{1}+i a_{2}\right), z=\frac{1}{\sqrt{2}}(x+i y), \partial=\frac{\partial}{\partial z}$.

[10] A. Polyakov and P. Wiegmann, Phys. Lett. 131B, 121 (1983)

[11] R. E. Gamboa Saravi, F. A. Schaposnik, and J. E. Solomin, Nucl. Phys. B185, 238 (1981).

[12] E. Witten, Comm. Math. Phys. 92, 455 (1984).

[13] T. Kugo and I. Ojima, Suppl. Progr. Theor. Phys. 66, 1 (1979).

[14] D. Karabali, Q. Han Park, H. Schnitzer, and Z. Yang, Phys. Lett. 216B, 307 (1989).

[15] P. Di Francesco, H. Saleur, and D. B. Zuber, Nucl. Phys. B29 [FS20], 527 (1987). 
[16] In what follows the arbitrary scale parameter $\mu$ in (16) will be conveniently chosen such as to conform with the results in the literature.

[17] V. G. Knizhnik and A. B. Zamolodchikov, Nucl. Phys. B247, 83 (1984).

[18] S. Naculich and H. Schnitzer, Nucl. Phys. B333, 583 (1990); B347, 687 (1990).

[19] B. Schroer and T. T. Truong, Nucl. Phys. B154, 125 (1979).

[20] L. P. Kadanoff and H. Ceva, Phys. Rev. B3, 3918 (1971).

[21] K. Gawedzki and A. Kupiainen, Nucl. Phys. B320, 624 (1989).

[22] R. Bariev, Sov. Phys. JETP 50, 613 (1979).

[23] B. McCoy and J. Perk, Phys. Rev. Lett. 44, 840 (1980).

[24] D. C. Cabra and K. D. Rothe, in preparation. 